\title{
Otto: An Autonomous School Bus System for Parents and Children
}

\begin{tabular}{|c|c|}
\hline Jackie Ayoub & Austin Kirchner \\
\hline University of Michigan & University of Michigan \\
\hline Dearborn MI, USA & Dearborn MI, USA \\
\hline Jyayoub@umich.edu & akirchne@umich.edu \\
\hline Brian Mason & Naira Tumanyan \\
\hline University of Michigan & University of Michigan \\
\hline Dearborn MI, USA & Dearborn MI, USA \\
\hline brmason@umich.edu & tumanyan@umich.edu \\
\hline Kamari Morse & Feng Zhou \\
\hline University of Michigan & University of Michigan \\
\hline Dearborn MI, USA & Dearborn MI, USA \\
\hline kamarim@umich.edu & fezhou@umich.edu \\
\hline
\end{tabular}

Permission to make digital or hard copies of part or all of this work for personal or classroom use is granted without fee provided that copies are not made or distributed for profit or commercial advantage and that copies bear this notice and the full citation on the first page. Copyrights for third-party components of this work must be honored. For all other uses, contact the Owner/Author.

CHI 2020 Extended Abstracts, April 25-30, 2020, Honolulu, HI, USA (c) 2020 Copyright is held by the owner/author(s).

ACM ISBN 978-1-4503-6819-3/20/04.

https://doi.org/10.1145/3334480.3382926

\begin{abstract}
Technological advances in autonomous transportation systems have brought them closer to road use.

However, little research is reported on children's behavior in autonomous buses (ABs) under real road conditions and on improving parents' trust in leaving their children alone in ABs. Thus, we aim to answer the research question: "How can we design ABs suitable for unaccompanied children so that the parents can trust them?" We conducted a study using a Wizard-of-Oz method to observe children's behavior and interview both parents and children to examine their needs in ABs. Using an affinity diagram, we grouped children's and parents' needs under the following categories: entertainment, communication, personal behavior, trust and desires. Using an iterative human-centered design process, we created an Otto system, a smartphone app for parents to communicate with their children and a tablet app for children to entertain during the ride.
\end{abstract}

\section{Author Keywords}

Autonomous bus; trust; human-centered design; child transportation.

\section{CCS Concepts}

- Human-centered computing $\rightarrow$ Interaction

design $\rightarrow$ Interaction design process and methods

$\rightarrow$ User centered design 


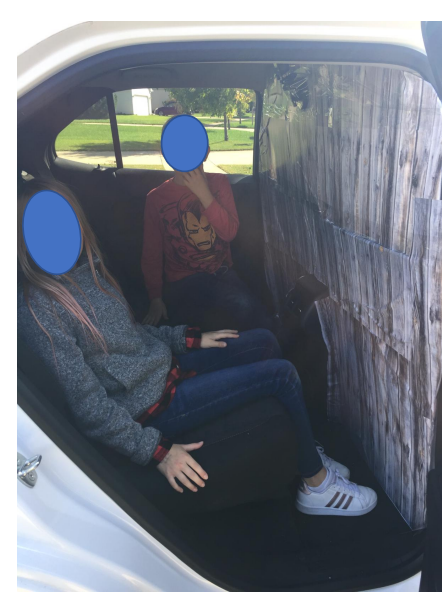

Figure 1: Interior setup of the vehicle.

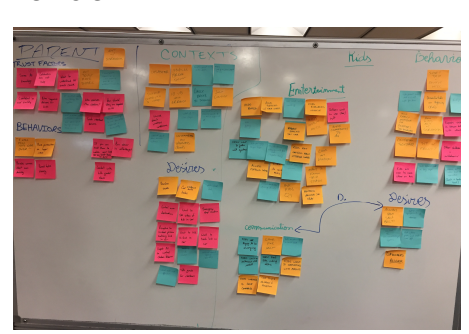

Figure 2: Affinity diagram.

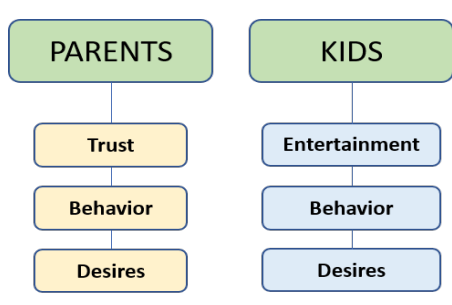

Figure 3: Resulting affinity diagram categories.

\section{Introduction}

One of the leading causes of deaths among 5-14 years old children is motor vehicle crashes [11]. Around 73\% of motor vehicle crash deaths in 2018 occurred when children were traveling as passengers [3]. With the potential to save children's lives and prevent collisions, autonomous vehicles (AVs) are promising. By eliminating accidents caused by human error, AVs can reduce traffic accidents by $90 \%$ [4]. In the 20402060s, SAE level 4 AVs will become common and affordable [12]. Therefore, it is safe to assume that all types of vehicles will be affected by the autonomous wave, including school buses. Thus, children who are using school buses today will eventually start using $A B s$ in the future as AVs become the norm. However, are parents and children ready for this change?

Shariff et al. [9] argued that the biggest roadblocks standing in the path of mass adoption of AVs may be psychological, not technological. This can be even harder when it comes to children as passengers traveling without adult supervision in the AVs, especially when considering sensitive issues, such as child safety, trust in automation, and laws and regulations around leaving children unattended in the vehicle for a period of time. Studies have shown that acceptance and trust have high potential impacts on user experience of AVs $[2,5,8,13]$. Models of human behavior and technology acceptance are based on the principle that people's perceptions and beliefs will determine their intentions, ultimately translating into actual behavior. Thus, it is critical to understand the major factors that shape intention and actual behavior [10]. In Technology Acceptance Model [3], perceived usefulness and perceived ease of use are identified as predictors of behavioral intentions. According to Lee and See [6], trust in AVs is defined as the attitude that the AV will help achieve one's goals in a situation characterized by uncertainty and vulnerability. Thus, an appropriate level of trust in AVs can translate into willingness to accept and use the technology [6]. As Shariff et al. [9] argued that the level of trust can determine how widely AVs would be adopted by users. Furthermore, considering that driving is a safety-critical activity, safety perception will be a key factor influencing the acceptance of AVs. If we cannot perceive sufficient safety from riding in $A V s$, it is expected that we will not accept or use them $[10,13]$.

\section{Research Objectives}

The topic of children in the autonomous setting has received little attention [7]. According to Anderson et al. [1], the safety features in AVs, existing policies, and the current infrastructure were impeding parents'

acceptance and trust in letting their children ride an AV. This highlights the need for more research to understand children's behavior in $A B s$ and to improve parents' trust in ABs. Therefore, the objectives of this research are 1) to understand children's behaviors, desires, and concerns about ABs, 2) to understand parents' feelings and concerns about letting their children ride in an $A B$, and 3 ) to design a system that accommodates both children's and parents' needs.

\section{Method and Data Collection}

In order to achieve our research objectives, we followed a human-centered design process suggested by Hasso Plattner Institute of Design at Stanford. The method consists of five phases - Empathize, Define, Ideate, Prototype, and Test. The five phases of the human-centered design process will be described in the following. 


\section{Persona 1- Poppy}

Characteristics: curious, troublemaker Goals: discover, have fun, make friends Hobbies: playing with friends

Frustrations: bored, stuck in seat

\section{Persona 2- Carl}

Characteristics: want company but no conversation

Goals: reunite with parents

Hobbies: Lego, Minecraft

Frustrations: bored, scared, lack of

control

\section{Persona 3- Bill and Karen}

Characteristics: always concerned about kids' safety

Goals: Kids' safety, monitor kids

Hobbies: Personal time

Frustrations: leaving kids alone,

managing work and family time

Figure 4: The three generated personas.

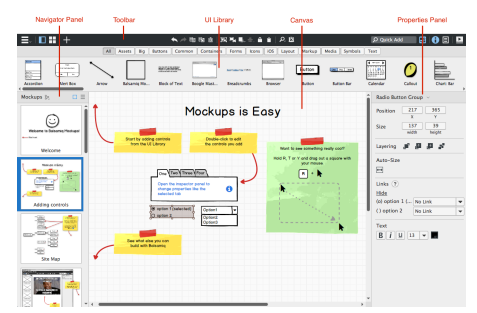

Figure 6: Balsamiq tool interface.

\section{Phase 1: Empathize}

We used a Wizard-of-Oz technique to observe children's behavior in an SUV where we added a foam board to separate the front and back seats as seen in Figure 1. A GoPro camera was installed in the middle of the board to record children's behavior during the ride. Field observations were conducted with four elementary school children and semi-structured interviews with three parents to study their perception on ABs. Four children ( 3 boys and 1 girl) were recruited in a neighborhood in Dearborn, MI and were compensated with cookies. The children where between the ages of 6 and 10 so that the illusion of an $A B$ could be more easily created and sustained. First, the children were explained that the SUV was operated by a hidden robot and that the robot could only recognize one command which was "robot stop". They should only issue it in case they were scared. The children were driven in the vehicle in their neighborhood for a 15-min ride. Second, after they watched videos of their children in the SUV ride, we interviewed three of their parents (two moms and one dad). We aimed to explore the following questions in the interviews, including a) how their children behave differently in the study from other rides with their parents, b) how much do they know regarding AVs, c) whether they are ready to let their children use them, and d) what features they would like to see in such systems.

\section{Phase 2: Define}

Based on the video and interview data in the empathy phase, we created an affinity diagram to identify the main categories (see Figure 2). We regrouped our postit notes multiple times until we settled upon our final diagram, which included two major categories: parents and children with three respective sub-themes, i.e., trust, behavior, and desires in the parents' category and entertainment, behavior, and desires in the children's category as shown in Figure 3. Using the results from the affinity diagram and the notes from the field studies, we began the persona generation process. In creating our personas, the team considered the following categories: key characteristics, goals, hobbies, and frustrations. These categories were chosen to understand the expectations and attitudes of children and parents towards $A B s$ and their needs in the design. As shown in Figure 4, we created two children personas and one parent persona.
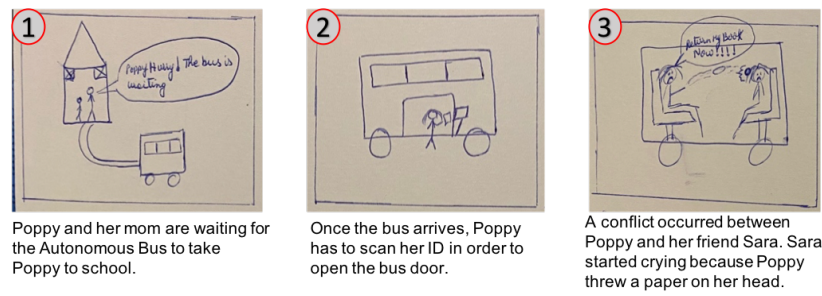

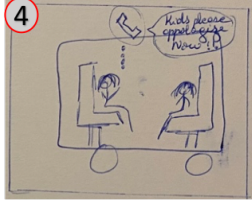
The agent responsible of
monitoring the kids called in an monitoring the kids cald

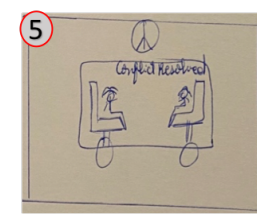

Problem resolved and
everyone is happy.

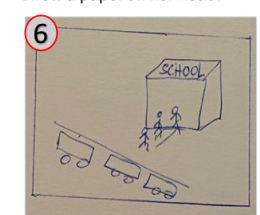

safe to school.
Figure 5: Storyboard scenario covering a route to school using an autonomous school bus.

After generating the personas, we created scenarios to help us identify what might happen during a ride and how our design could handle these scenarios. We created two scenarios. In one of the scenarios, Carl was using an $A B s$ with a few key activities that these types of users might perform to understand what functions 


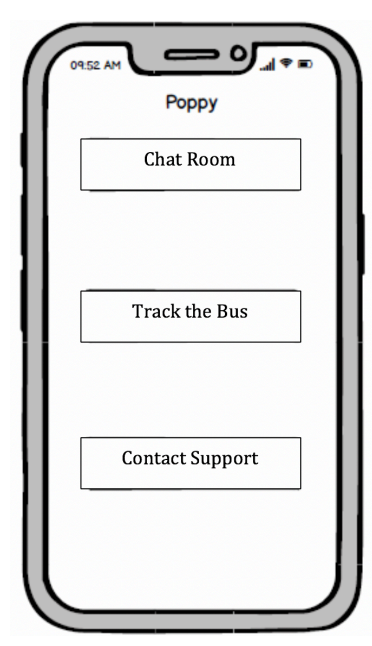

Figure 7: OttoParent screen.

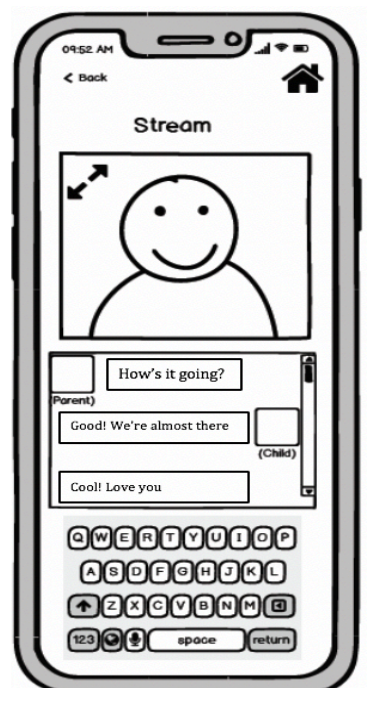

Figure 8: OttoParent text/video chat screen. and features our system should support. These activities included getting on the bus, calling a parent, expressing needs for emotional support, listening to his favorite book, and safely arriving at his destination (communicated to his parents by a confirmation message). The second scenario is shown in Figure 5 which describes a conflict happening with Poppy during the ride. The personas and scenarios help us to define our design challenge as: "How can we design ABs suitable for unaccompanied children so that the parents can trust them with their children?".

\section{Phase 3: Ideate}

We combined brainstorming with scribble sketching to generate ideas. By sharing stories from the field studies, we practiced group scribble sketching on a white board. Then, each member selected the top five ideas from the board that covered the themes we gathered through the affinity diagram and the needs from the personas and scenarios to move to the next round for brainstorming. During this round of brainstorming, we started to select and combine ideas into full system solutions. Every solution that we finally agreed on was thoroughly vetted by our personas. After completing our scribble sketching and brainstorming, then selecting and combining ideas into system solutions, we decided to place the key elements in a smartphone/tablet app, named Otto system, for parents and children to use in $A B$ rides.

Phase 4-5: Prototype and Test

After sketching the ideas selected for the children and parent interfaces, we chose to proceed with a lowfidelity vertical prototype using a wireframing program called Balsamiq (see Figure 6). The first prototype was brought to a co-design session with parents and children where we asked them to think aloud about how to navigate through the app. Then, we offered printouts to the participants and asked them to sketch ideas to improve the prototype. Among think aloud interview, and co-design sessions, we gathered feedback used to iteratively improve the system. In this process, it is critical that the OttoParent interface instill trust in parents while providing simple functionality. The system enables parents to monitor their children inside the vehicle, contact school administration and emergency personnel through the app, communicate with the children while in the $A B$, and provide updates about the status of pick-up and drop-off, physical location of the car, route changes, and other relevant updates. The parents must also feel that the system can take care of their children if they are unavailable and they must feel that they can provide feedback which will improve the future ride experience. This resulted in a fairly simple structure of the parent app. First, the parents are greeted by a title page with log-in/registration options. Then, the parents are prompted to create an account and populate it with their information and their children's information. The parents add information for their children, which later appear as profiles for them. Within the children's profiles, the parents have options to chat with them, track the bus, and contact support (see Figure 7). The chat screen has both text and video chat options (see Figure 8). The bus tracking screen shows the location on a map as well as time remaining and percentage complete. The "Contact Support" screen offers both emergency and problem reporting options. The emergency option connects the parents directly with authorities while the "Report a Problem" option allows them to enter text feedback. Lastly, push notifications 


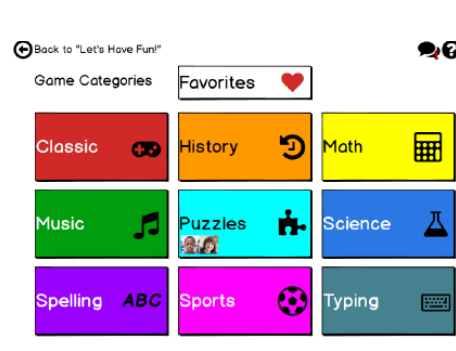

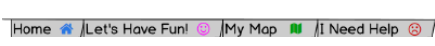

Figure 11: OttoKids "Games" categories.

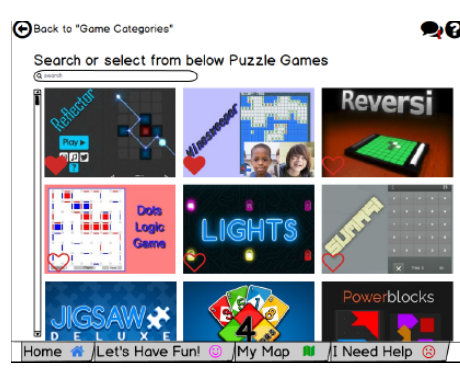

Figure 12: OttoKids "Games" selections.

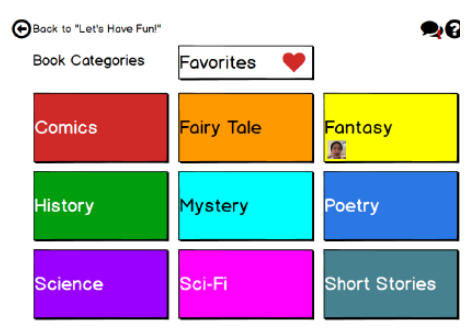

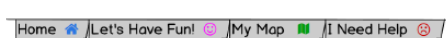

Figure 13: OttoKids "Book" categories. offer updates outside the app for events, such as confirmation on children's arrival.

The OttoKids app must entertain children while providing communication options to connect with parents or with emergency personnel. If children are not entertained, they are more likely to cause trouble with other children or experience negative emotions, such as fear and boredom (e.g., "Oh my god! What if the police pull the car over and there is no driver?"). Communication with parents and emergency personnel needs to be easily accessible whenever children experienced problems. Entertainment means many things to children, such as games, books, videos, watching the trip progress, and talking to someone. Thus, the system attempts to provide options for these major categories while encouraging interaction with others in the vehicle. To bolster parental support, the entertainment options must be age-appropriate and developmental in nature, adding homework as a possible activity. An avatar named Otto guides children through these options, acting as a companion and providing a communication pathway. When a child enters the vehicle, he or she will be greeted by the homepage which includes Otto and a visual representation of the other bus riders as well as navigation options (see Figure 9). The child can talk to Otto directly to select options or click on the screen. Clicking on the other bus riders shows what they are doing and allows the child to join them in that activity. Selecting "Let's Have Fun" takes the child to a screen where they can select among games, books, videos, and homework and see what others are doing (see Figure 10). These options all have subpages that show different categories. These categories open up to tilebased lists showing the available options (see Figures
$11,12,13,14)$. "My Map" allows the child to view the bus location on a map with the route and destination displayed as well as a trip progress indicator (see Figure 15). "I Need Help" gives the child options to contact his/her school, parents, or emergency personnel (see Figure 16). The emergency option requires two clicks to reduce false alarms. Clicking on the persistent comment option in the top right will take the child to the parent chat page while clicking on the question mark will take them to the help page.

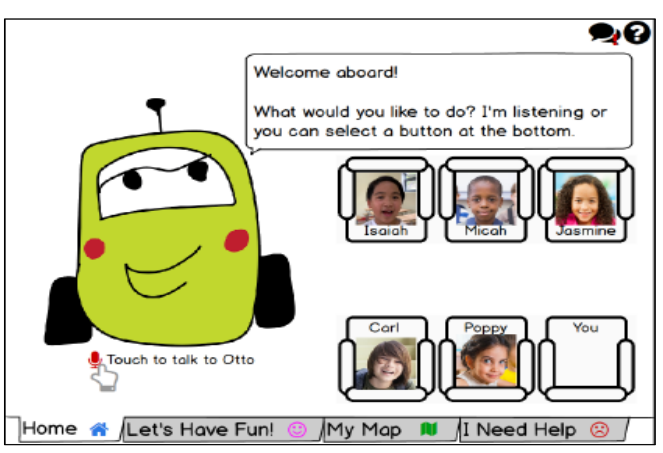

Figure 9: OttoKids entry screen.

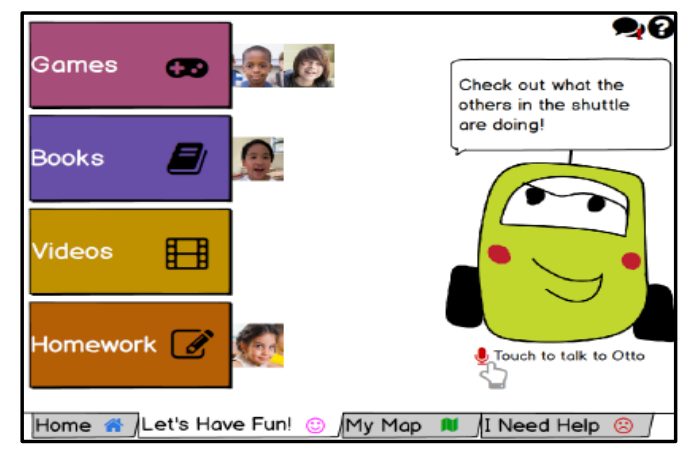

Figure 10: OttoKids "Let's have fun" screen. 


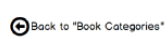

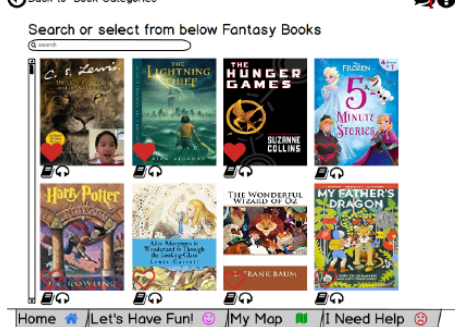

Figure 14: OttoKids "Book" selection.

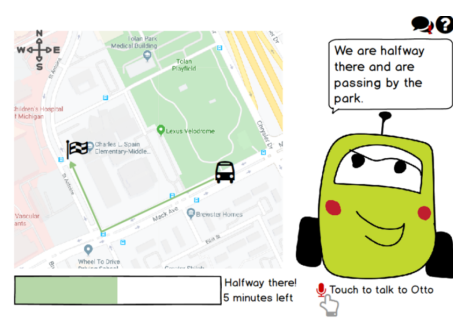

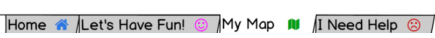

Figure 15: OttoKids "My Map" screen.

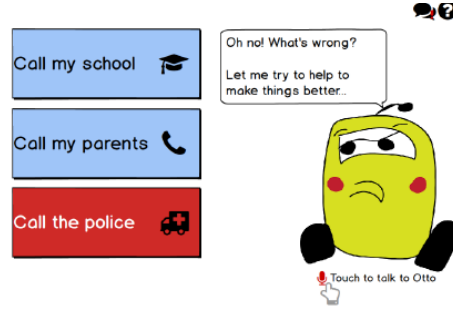

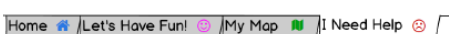

Figure 16: OttoKids "I Need Help" screen.
As a summary, the Otto system must keep the child entertained and safe while instilling trust in parents. It requires approval from school administrators. There are three main digital touchpoints for the product: a) OttoParent - An app for parents to summon and track the bus and their children, b) OttoKids - An onboard system for children to entertain, instruct, and, in case of emergency, contact the proper personnel, and c) Ingress/Egress - A hardware/software solution that ensures that the correct child is being picked up and dropped off to the correct people at the correct place. Updates for OttoKids will be required before the start of each semester to reflect all the changes in the libraries (books/games/videos). In addition, the vehicle will need to be able to know who is getting in and out of the $A B$ by ID verification using either a phone/card scan, a camera facial recognition app, a biometric scan, or an RFID reader.

\section{Discussions and Conclusion}

Based on our field studies, both stakeholders had different primary concerns: the parents wanted to make sure their children were safe and the children wanted to avoid boredom and fear. However, there were concerns by both parties about what would happen in different emergencies of varying severity. The solution would need to cover a broad range of responses for a broad range of situations.

Our findings ultimately came down to a series of requirements based on our study and literature. The children should be entertained while remaining safely secured in their seats. The best way to achieve this is with stowable edutainment tablets which also allow for contact with Otto support staff, parents, the school, and emergency services if necessary. The parents should be able to track the location and activity of the children as well as monitor and communicate with them via video. With the Otto system, we addressed the issues of trust, safety, and usefulness, making ABs suitable for children. Therefore, it is possible to increase parents' trust in ABs in the future through a human-centered design process.

We also recognized the limitations of our study. First, our recruited participants (both parents and children) had little to no experience with ABs, and that they were mostly mentally imagining the experience of riding the ABs in our study, despite the fact that the children in the simulated AV with the Wizard-of-Oz method believed that it was an AV. Moreover, we used an SUV rather than a school bus for simulation purpose, which could potentially reduce the generalizability of the results to school buses. Second, this study included only four elementary school children in order for them to believe that it was an AV. In the future, more research should be conducted for other age groups in an AV with the Otto system on for testing purposes. Third, a potentially fertile research could be done to clarify children's conception of the appropriate level of help. There was a degree of ambiguity around who should be contacted for what type of

emergency/behavior, although we obtained valuable feedback during the prototyping and testing phase.

Similarly, there was a degree of ambiguity on how best to direct the children to the appropriate response.

\section{References}

[1] James M. Anderson, Kalra Nidhi, Karlyn D. Stanley, Paul Sorensen, Constantine Samaras, and Oluwatobi A. Oluwatola. 2014. Autonomous Vehicle Technology: A Guide for Policymakers. Rand Corporation. 
[2] Jackie Ayoub, Feng Zhou, Shan Bao, and X. Jessie Yang. 2019. From Manual Driving to Automated Driving. Proceeding of the 11th International Conference on Automated User Interfaces and Interactive Vehicular Applications-

AutomotiveUI'19.

https://doi.org/10.1145/3342197.3344529

[3] Fred D. Davis. 1989. Perceived Usefulness, Perceived Ease of Use, and User Acceptance of Information Technology. The Mississippi quarterly 13, 3: 319-340.

[4] Janet Fleetwood. 2017. Public Health, Ethics, and Autonomous Vehicles. American journal of public health 107, 4: 532.

[5] Anna-Katharina Frison, Philipp Wintersberger, Andreas Riener, Clemens Schartmüller, Linda $\mathrm{Ng}$ Boyle, Erika Miller, and Klemens Weigl. 2019. In UX We Trust: Investigation of Aesthetics and Usability of Driver-Vehicle Interfaces and Their Impact on the Perception of Automated Driving. In Proceedings of the $2019 \mathrm{CHI}$ Conference on Human Factors in Computing Systems (CHI '19), Association for Computing Machinery, New York, NY, USA, Paper 144, 1-13.

DOI:https://doi.org/10.1145/3290605.3300374

[6] John D. Lee and Katrina A. See. 2004. Trust in Automation: Designing for Appropriate Reliance. Human Factors: The Journal of the Human Factors and Ergonomics Society 46, 50-80. https://doi.org/10.1518/hfes.46.1.50.30392

[7] Yi-Ching Lee and Jessica H. Mirman. 2018. Parents' perspectives on using autonomous vehicles to enhance children's mobility.

Transportation Research Part C: Emerging Technologies 96, 415-431.

https://doi.org/10.1016/j.trc.2018.10.001

[8] Andreea I. Niculescu, Alan Dix, and Kheng Hui Yeo. 2017. Are You Ready for a Drive? User Perspectives on Autonomous Vehicles. In
Proceedings of the $2017 \mathrm{CHI}$ Conference Extended Abstracts on Human Factors in Computing

Systems (CHI EA '17). Association for Computing Machinery, New York, NY, USA, 2810-2817. DOI:https://doi.org/10.1145/3027063.3053182

[9] Azim Shariff, Jean-François Bonnefon, and Iyad Rahwan. 2017. Psychological roadblocks to the adoption of self-driving vehicles. Nature Human Behaviour 1, 694-696. https://doi.org/10.1038/s41562-017-0202-6

[10] Zhigang Xu, Kaifan Zhang, Haigen Min, Zhen Wang, Xiangmo Zhao, and Peng Liu. 2018. What drives people to accept automated vehicles? Findings from a field experiment. Transportation Research Part C: Emerging Technologies 95, 320334. https://doi.org/10.1016/j.trc.2018.07.024

[11] Ten Leading Causes of Death and Injury PDFs|Injury Center|CDC. Retrieved December 8, 2019 from

https://www.cdc.gov/injury/wisqars/LeadingCause s.html

[12] Todd Litman. 2015. Autonomous vehicle implementation predictions: Implications for transport planning. Retrieved from https://trid.trb.org/view/1338043

[13] Feng Zhou, X. Jessie Yang, and Xin Zhang. 2019. Takeover Transition in Autonomous Vehicles: A YouTube Study. International Journal of HumanComputer Interaction, 36, 3: 295-306. https://doi.org/10.1080/10447318.2019.1634317 